\title{
les argilites surconsolidées du Domérien de Nancy : relations entre la consolidation, la diagenèse et quelques propriétés mécaniques
}

\author{
par \\ Jean-Pierre Troalen \\ Assistant \\ Institut National Polytechnique de Lorraine, E.N.S. G., \\ Laboratoire de Mécanique et d'Hydraulique des Sols et des Roches
}

\section{Introduction}

Les argilites du Domérien, d'épaisseur maximale $82 \mathrm{~m}$, se rencontrent dans la région de Nancy, soit à l'affleurement, soit lors de fouilles ou de sondages. Elles peuvent se trouver sous une couverture sédimentaire pouvant atteindre le Bajocien ou sous des formations superficielles souvent alluviales.

Ces argilites sont des matériaux surconsolidés et leur état actuel est le résultat des actions successives des phénomènes diagenétiques, de la consolidation et des processus d'érosion ultérieurs.

Les recherches effectuées jusqu'à présent ont abordé aussi bien l'aspect sédimentologique (1) que le comportement mécanique des argilites (9), (8), (4), (6) et (7). Elles ont permis de dégager diverses méthodes d'identification et de mesure des propriètés physiques ou mécaniques. Cependant, les corrélations entre ces propriétés restent peu nombreuses. D'autre part, dans le cas particulier des argilites surconsolidées, I'homogénéité apparente des matériaux (nature minéralogique, identification géotechnique) ne permet pas de lui corréler un comportement mécanique.

Des essais de désagrégation, complétés par l'étude des textures au microscope électronique à balayage, mettent en évidence le diagenèse, des différentes liaisons acquises par le matériau durant sa diagénèse, permettent d'estimer les énergies de liaison correspondantes et la prévision de certains types de comportement mécanique.

Les liaisons acquises durant la diagenèse peuvent être classées en deux familles. Les forces d'attraction moléculaires (forces de Van der Waals), les liaisons hydrogènes ou oxygènes et celles dues à l'introduction d'ions dans la structure forment la première famille. Toutes ces liaisons sont sensibles à l'eau qui les détruit ou les neutralise. Elles caractérisent les matériaux du type " sol ". La seconde famille est plus complexe et est constituée par les liaisons interparticulaires. Ce sont les liaisons qui s'opposent aux contraintes dévelop- pées par des minéraux gonflants, par les phénomènes de décompression ou d'altération météorique et aux sollicitations classiques des essais de géotechnique (limites d'Atterberg par exemple). L'expression «liaisons interparticulaires " paraît préférable à celle dite "liaisons texturales" citée par Le Roux (3). En effet, "liaisons texturales" caractérise des matériaux type "roche " et est souvent assimilée au terme "ciment . Les liaisons interparticulaires désignent donc des liaisons caractéristiques des matériaux argileux surconsolidés, intermédiaires entre un "sol $*$ et une "roche".

Le carbonate de calcium (micrite), fréquent dans les argilites du Domérien, apparaît alors comme une forme de liaison interparticulaire et non comme un ciment continu.

Dans l'exposé qui va suivre, l'essentiel du raisonnement portera sur les liaisons interparticulaires, essentiellement seules responsables des anomalies observées dans le comportement mécanique des argilites du Domérien.

2 Diagenèse, consolidation, phénomènes ultérieurs et comportement mécanique

D'origine marine et initialement plastiques, les argilites du Domérien ont été progressivement consolidées sous des charges croissantes pour atteindre une pression de consolidation maximale. L'épaisseur des surcharges réellement imposées est difficile à calculer. Ces argilites ont fini de se déposer il y a environ 180 millions d'années et le modelé actuel de la région lorraine est daté du Plio-quaternaire. Malgré l'imprécision des reconstitutions paléogéographiques, le recouvrement peut être estimé à plusieurs centaines de mètres (6). La pression des terrains de recouvrement s'est maintenue au moins jusqu'au Pliocène et des processus physico-chimiques (diagenèse) se sont produits à l'intérieur des argilites. II en résulte une déformation des feuillets argileux avec emboitement 
$w, n, w_{1}, w_{p}, I_{p}(\%)$

A : pourcentage d'argiles obtenu par' la méthode des résines échangeuses d'ions

$\mathrm{CaCO}_{3}$ : pourcentage par dosage par complexométrie à l'E. G. T. A. (acide Éthylène Glycol) bis (amino 2 ètyléther) N, $\mathrm{N}, \mathrm{N}^{\prime}, \mathrm{N}^{\prime}$ - tétracétique)

E. L.I. : énergie de liaison interparticulaire

Surface spécifique $\left(\mathrm{m}^{2} / \mathrm{g}\right)$ obtenue par la méthode B.E.T. (Brunauer, Emmet et Teller)

\begin{tabular}{|c|c|c|c|c|c|c|c|c|c|c|c|c|c|c|}
\hline \multirow[b]{3}{*}{ Sommet } & \multirow{3}{*}{$\begin{array}{c}\text { Niveau } \\
5\end{array}$} & \multirow{3}{*}{$\begin{array}{c}\text { Classification } \\
\text { silto-argilite } \\
\text { calcaire }\end{array}$} & \multicolumn{5}{|c|}{ Poids volumiques } & \multicolumn{3}{|c|}{ Limites d'Atterberg } & \multirow[b]{2}{*}{ A } & \multirow{3}{*}{$\begin{array}{c}\mathrm{CaCO}_{3} \\
11.9\end{array}$} & \multirow{3}{*}{$\begin{array}{l}\text { E. L.I. } \\
0.76\end{array}$} & \multirow{2}{*}{$\begin{array}{l}\text { Surface } \\
\text { spécifique }\end{array}$} \\
\hline & & & w & e & $n$ & $\gamma_{n}$ & $\gamma_{d}$ & $w_{1}$ & $w_{D}$ & $I_{p}$ & & & & \\
\hline & & & 10.4 & 0,223 & 18,2 & 22,1 & 20,9 & 38,5 & 20 & 18,5 & 31.6 & & & 20,1 \\
\hline \multirow{3}{*}{$35 \mathrm{~m}$} & 4 & $\begin{array}{c}\text { silto-argilite } \\
\text { calcaire }\end{array}$ & 9,4 & 0,229 & 18,6 & 21,9 & 20,8 & 42 & 21,9 & 20,1 & 36,2 & 5,4 & 0,68 & 25 \\
\hline & 3 & silto-argilite & 11,2 & 0,288 & 22,3 & 21,3 & 19,2 & 48,5 & 22,4 & 26,6 & 45 & 2,8 & 0,82 & 25 \\
\hline & 2 & $\begin{array}{l}\text { silto-argilite } \\
\text { calcaire }\end{array}$ & 12,8 & 0,258 & 20,5 & 22 & 20 & 50,0 & 22,7 & 27,3 & 4,73 & 11,7 & 0,31 & 18 \\
\hline base & 1 & $\begin{array}{l}\text { silto-argilite } \\
\text { calcaire }\end{array}$ & 9.6 & 0.196 & 16,3 & 21.7 & 20.6 & 55 & 25.6 & 29.4 & 58,7 & 6,3 & 0,85 & 29,7 \\
\hline
\end{tabular}

des surfaces de contact et il y a eu création de liaisons type "sol" et interparticulaires. A ces phénomènes s'est ajoutée une liaison partielle par le carbonate de calcium, sous forme de micrite (précipitation) ou de recristallisation. II en résulte, dans les argilites, une cohésion qui va donc dépendre de la pression de consolidation atteinte, de la nature des minéraux constitutifs, de la pression interstitielle régnante et du laps de temps écoulé. La cohésion ainsi acquise est plus ou moins renforcée par la présence de carbonates. Les argilites acquièrent ainsi une structure beaucoup plus raide et par là même plus fragile (2).

La phase d'érosion majeure (Plio-quaternaire) a pour résultat de provoquer une décompression progressive des argilites (déchargement). Les argilites ont alors une tendance au gonflement mais cette expansion se trouve freinee par les liaisons interparticulaires acquises pendant la diagenèse. Si le déchargement devient important (argilites à l'affleurement ou sous une faible couverture alluviale), les liaisons interparticulaires peuvent être brisées en partie ou en totalité. Cependant, on constate que le même déchargement ne produit pas obligatoirement un même effet de décompression dans les argilites.

II en résulte que la prévision du comportement mécanique et des propriétés afférentes reste assez aléatoire, même si l'identification des matériaux a abouti à l'homogénéité et à l'identité de leur nature (8).

II devient nécessaire d'effectuer préalablement à létude des propriétés mécaniques des essais de caractérisation complémentaires.

Des essais récents sur le comportement sous chargement cyclique des argilites raides et fissurées du Domérien de Nancy ont montré que les réseaux de discontinuités potentiels ou exprimés n'avaient pas ou peu d'influence sur les paramètres de rupture. Seules les microfissures parallèles à l'axe des éprouvettes prélevées horizontalement (parallèlement à la surface $S_{o}$ du plan de stratification) semblent influencer le développement du plan de cisaillement principal (4), (6) et (7).

L'orientation référée des, éprouvettes d'essai, la description des réseaux de discontinuités (dynamiques ou sédimentaires), entreprises par Vigouroux (9), Troalen (8) et Plaza Nieto (4), peuvent être considérées comme intéressantes sur le plan descriptif mais elles sont - paradoxalement - inutiles vis-à-vis d'une tentative de corrélation avec les paramètres de la rupture : les essais de compression triaxiale " homogénéisent * les éprouvettes d'argilite.

En conséquence, il apparaît aujourd'hui préférable d'orienter l'étude préliminaire sur la caractérisation des liaisons interparticulaires acquises pendant la diagenèse par les argilites et d'essayer d'estimer l'énergie de liaison résultante (état actuel). Les hypothèses de travail proposées par Troalen (8) sont reprises et complétées. La méthode d'identification globale est cependant conservée.

\section{Identification physique et géotechnique des argilites}

Les matériaux étudiés proviennent de la carrière des G.M.E. Tuiles de Jeandelaincourt (54) où les 35 derniers mètres des "argiles à Amalthées" auct. (puissance totale $82 \mathrm{~m}$ ) forment le front de taille. Ils sont recouverts par $10 \mathrm{~m}$ de "Grès médioliasiques " auct. qui terminent le sous-étage Domérien de la région de Nancy.

Du point de vue de la classification, les matériaux 
ètudiés sont des "silto-argilites" et des "silto-argilites calcaires". Le pourcentage total en $\mathrm{CaCO}_{3}$ n'excède pas $12 \%$

\section{Argilites surconsolidées (Domérien) : \\ Constituants :}

Minéraux argileux : 50 à $60 \%$ de la roche décarbonatée; illite dominante, kaolinite, chlorite, quelques édifices interstratifiés.

Sablons : 3 à $12 \%$ de la roche décarbonatée.

Silts (quartz-micas) : 40 à $50 \%$ de l'insoluble.

Fragments biogènes $\left(\mathrm{CaCO}_{3}\right): 3$ à $5 \%$ de la roche totale; taille moyenne $80 \mu \mathrm{m}$; peuvent dépasser $250 \mu \mathrm{m}$.

Pyrite, oxydes de fer : 2 à $3 \%$ de la roche totale; grains ou en "colonisation" de fragments biogènes.

Matière organique: $1 \%$ en moyenne.

Couleur état sec : $5 \mathrm{G} 6 / 1$ gris verdâtre.

Couleur état humide : $5 \mathrm{G} 4 / 1$ gris verdâtre foncé (selon le code Mundsell des couleurs).

A l'état naturel, les matériaux apparaissent en surface soit altérés et plastiques, soit indurés et fragmentés. Après décapage superficiel, les argilites sont massives et saines; cependant, une modification même légère de leur teneur en eau révèle ou efface les réseaux de discontinuités: destruction ou neutralisation des liaisons type "sol",

Du point de vue géotechnique, les argilites ont èté caractérisées par les paramètres physiques suivants

teneur en eau naturelle $(w)$.

porosité ( $n$ ),

indice des vides (e),

poids volumiques $(\gamma)$,

limites d'Atterberg $\left(w_{1}, w_{p}, I_{p}\right)$.

Les résultats moyens obtenus sur les argilites indurées saines de la carrière de Jeandelaincourt sont reportés dans le tableau $I$ et caractérisent les cinq niveaux définis pour les argilites à Amalthées par Allouc (1) et Troalen (8).

Dans le tableau I ont été ajoutés les pourcentages en argiles, en $\mathrm{CaCO}_{3}$, les surfaces spécifiques et les énergies de liaison résultantes.

Les résultats donnés dans le tableau I montrent l'homogénéité relative des argilites du Domérien. Le paramètre e caractérise l'évolution normale liée au processus de la consolidation puisque cet indice des vides diminue avec l'augmentation de profondeur. Les paramètres $w, n$ et $\gamma$ sont à relier aux caractéristiques minéralogiques et aux pourcentages correspondants en argiles $(A)$ et en carbonate de calcium. Les limites d'Atterberg sont effectuées sur un matériau remanié (mortier: particules inférieures à $400 \mu \mathrm{m}$ ) et déstructuré. Elles sont liées, d'une part à la phase argileuse, donc aux surfaces spécifiques et, d'autre part, aux pourcentages en $\mathrm{CaCO}_{3}$ qui peuvent jouer un rôle cimentant.

N.B. : Ces résultats sont en accord avec ceux obtenus par Troalen (8) sur l'ensemble régional des argilites du Domérien.

\section{Essais au laboratoire : cycles séchage/mouillage, énergie de liaison interparticulaire}

II a été prélevé 60 échantillons d'environ $2 \mathrm{~kg}$ chaque après décapage superficiel (argilites massives) répartis dans les cinq niveaux de la carrière de Jeandelaincourt (échantillonnage horizontal et vertical).

Chaque échantillon (bloc ou motte) apparaissait massif à l'état naturel. Le bloc est fractionné en deux morceaux d'1 kg. Sur un lot est effectuée la caractérisation physique (paragraphe 3 ); sur le deuxième lot sont réalisés des cycles mouillage/séchage.

\subsection{Cycles mouillage/séchage}

L'influence du traitement eau/température a déjà été abordée pour ces argilites par Thomas (5) et Troalen (8) et les résultats ont conduit au choix d'un essai constitué par deux cycles

immersion dans eau distillée $48 \mathrm{~h}$ passage à l'étuve à $105^{\circ} \mathrm{C} 48 \mathrm{~h}$

$\times 2$.

L'échantillon est immergé dans un cristallisoir remplí d'eau distillée. Il y est maintenu $48 \mathrm{~h}$ à la température de $20^{\circ} \mathrm{C}$. II est effectué ensuite un siphonnage de l'eau (sans élimination des particules fines), puis l'échantillon (avec son cristallisoir) est placé dans une étuve et maintenu à $105^{\circ} \mathrm{C}$ pendant $48 \mathrm{~h}$. Le cycle est renouvelé une fois. Après refroidissement à température ambiante, l'échantillon est tamisé à sec.

La répétition des cycles mouillage/séchage provoque une fragmentation de l'échantillon. Les liaisons type " sol "sont - en général - détruites ou neutralisées par ce traitement. Les liaisons interparticulaires à faible énergie de liaison sont également rompues.

Si l'on part du principe que "plus les liaisons interparticulaires sont nombreuses, moins de particules élémentaires seront libérées, et réciproquement ", il est alors simple de pratiquer une analyse granulométrique de l'échantillon après les cycles mouillage/séchage.

On appelle FG la fraction grossière (éléments de dimension supérieure à $80 \mu \mathrm{m}$ ) et FF la fraction fine de l'échantillon (éléments de dimension inférieure à $80 \mu \mathrm{m})$. Par définition, $F G+F F=100 \%$.

La fraction grossière correspond aux élèments agrégés par une énergie de liaison interparticulaire forte. II est possible de corriger ce pourcentage en déduisant de FG les éventuels \% relatifs à des bioclasts ou aux grains de pyrite éventuels. La fraction fine correspond aux éléments libérés par rupture des liaisons. Le \% en bioclasts est en général inférieur à $0,5 \%(8)$.

Si maintenant on désigne par E.L.l. (Énergie de Liaison Interparticulaire) le paramètre égal à $\frac{F G}{100}$, on obtient :

- pour E.L.I. $=1, F G=100 \%, F F=0 \%$; - pour E.L.I. =0, FG = 0\%, FF $=100 \%$.

Eli varie donc de 0 à 1. L'avantage de ce raisonnement est qu'il s'applique à tous les matériaux argileux surconsolidés.

Si l'on veut maintenant classer les énergies de liaison en fonction de leur intensité, on peut écrire la classification suivante :
Valeur de E. L.I.
1 à 0.75
0,75 à 0,55
0,55 à 0,45
0,45 à 0,25
0,25 à 0

Dénomination (intensité) forte assez forte moyenne assez faible faible 
Les résultats globaux sur les argilites du Domérien de Jeandelaincourt figurent dans le tableau I. En utilisant la classification énoncée précédemment et les résultats du paragraphe 4.3.2, on obtient (tableau II) :

Tableau II - E. L.I. et type de liaisons interparticulaires des argilites surconsolidẻes du Domérien de Jeandelaincourt

$\begin{array}{cccc}\text { Argilites } & \text { Valeur de E.L.I. } & \text { Intensité } & \text { Type de liaison majeur } \\ \text { Niveau } 5 & 0,76 & \text { forte } & \text { A/A, A/M } \\ \text { Niveau 4 } & 0,68 & \text { assez forte } & \text { A/A, A/M } \\ \text { Niveau 3 } & 0,82 & \text { forte } & A / A, A / M \\ \text { Niveau 2 } & 0,31 & \text { assez faible } & \text { A/A, A/mn, A/pc, A/B } \\ \text { Niveau 1 } & 0,85 & \text { forte } & A / M, A / A \text { majeur }\end{array}$

Ces résultats sont intéressants mais ne permettent pas de définir les types de liaisons interparticulaires. A ce stade de l'analyse, seul est estimé le pourcentage global du nombre de liaisons interparticulaires.

Commentons tout d'abord ces premiers résultats.

\subsection{Résultats partiels}

Une première analyse montre que les cinq niveaux d'argilite présentent des E. L.I. voisines à l'exception du niveau 2.

L'acquisition des liaisons interparticulaires dépend de I'histoire du matériau, c'est-à-dire de sa diagenèse, de la pression maximale de consolidation, de la pression interstitielle, des minéraux constitutifs, du temps et des agents cimentants éventuels.

D'autre part, à l'état actuel dans le prélèvement, les phases de déchargement ont décompressé en partie les matériaux. En conséquence, les énergies de liaison interparticulaire mesurées sont des énergies ultimes qui traduisent l'état actuel des matériaux, c'est-à-dire au moment de leur prélévement.

$\mathrm{Si}$, comme on l'a noté précédemment, l'identification a conclu à une quasi-identitè physique et minéralogique des matériaux étudiés, il apparaît avec les cycles mouillage/séchage une anisotropie des comportements mécaniques. II convient donc d'analyser d'une façon plus intime les textures et les types de liaisons interparticulaires de ces matériaux, c'est-à-dire la répartition spatiale et la nature des constituants.

Les divers échantillons ont été observés au microscope électronique à balayage (M.E.B.) après métallisation des pastilles à l'or-palladium. Le microscope polarisant à transmission a un pouvoir sèparateur insuffisant (les particules argileuses se trouvent superposées dans une lame mince de $30 \mu \mathrm{m}$ d'épaisseur).

\subsection{Analyse au M.E. B. des argilites}

Les observations ont essentiellement porté sur les textures, les liaisons interparticulaires et la distribution de cette répartition spatiale des minéraux constitutifs dans les cinq niveaux repères des argilites du Domérien.

\subsubsection{Les textures}

Deux principaux types ont été observés. Le premier est une texture d'aspect lité (photographie 1) et il correspond aux argilites des niveaux 1, 2, 3 et 4 . Les feuillets d'illite sont déformés et emboîtés les uns dans les autres. La déformation est accentuée au contact d'un fragment biogène. Les " trous " observés correspondent sans doute à l'arrachement de petits grains de quartz (silts) ou de grains pyriteux (photographies 3 et 4). Le second type présente une texture plus massive due à la cimentation partielle des feuillets argileux par le carbonate de calcium (photographie 2). Cette seconde texture n'est fondamentalement pas très différente du premier type.

Localement, il apparaît dans la texture litée du niveau 2 un sous-type textural qui montre alors des amas minéraux, véritables "rosaces " ou « nids" intercalés dans les feuillets encaissants (illite) (photographie 6). L'analyse minéralogique des amas conclut à des minéraux type interstratifiés montmorillonite/illite. Ceci confirme ce qui était apparu aux analyses par diffractométrie des rayons $X(8)$. De ce fait, on peut raisonnablement envisager que les minéraux interstratifiés type illite/montmorillonite sont diffus dans les argilites des niveaux $1,3,4$ et 5 et en gîte d'accumulation dans le niveau 2. Des amas de ce type ont déjà été observés et décrits par Le Roux (3) dans les marnes de Lodève.

\subsubsection{Les liaisons interparticulaires}

L'observation au M.E.B. permet d'envisager certains comportements des argilites surconsolidées en présence d'eau et met en évidence différents types de liaisons interparticulaires et de liaisons type "sol».

Par sliaisons interparticulaires", il faut entendre toutes les liaisons qui s'opposent aux efforts et contraintes développés par les processus de décompression, les minéraux gonflants et les actions mécaniques lors des essais classiques de Mécanique des Sols (limites d'Atterberg, par exemple). Ces liaisons interparticulaires possèdent une énergie de liaison (E.L. I.) plus forte que celle des liaisons type "sol". 


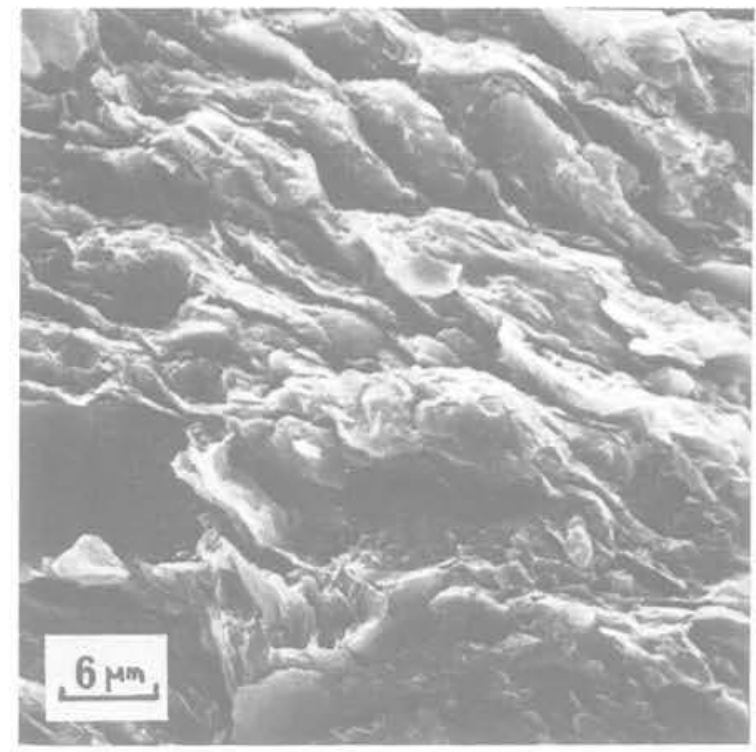

Photo 1

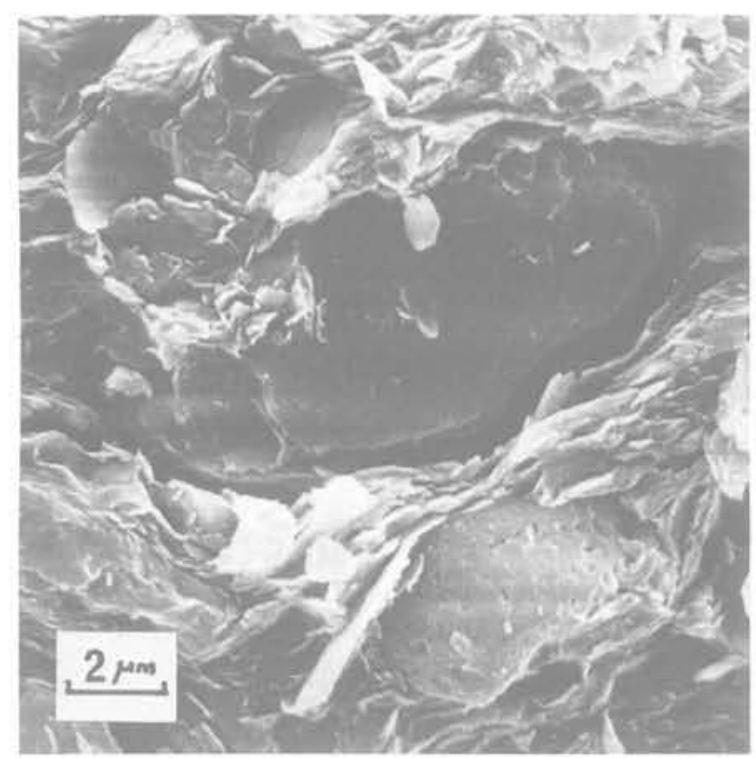

Photo 3

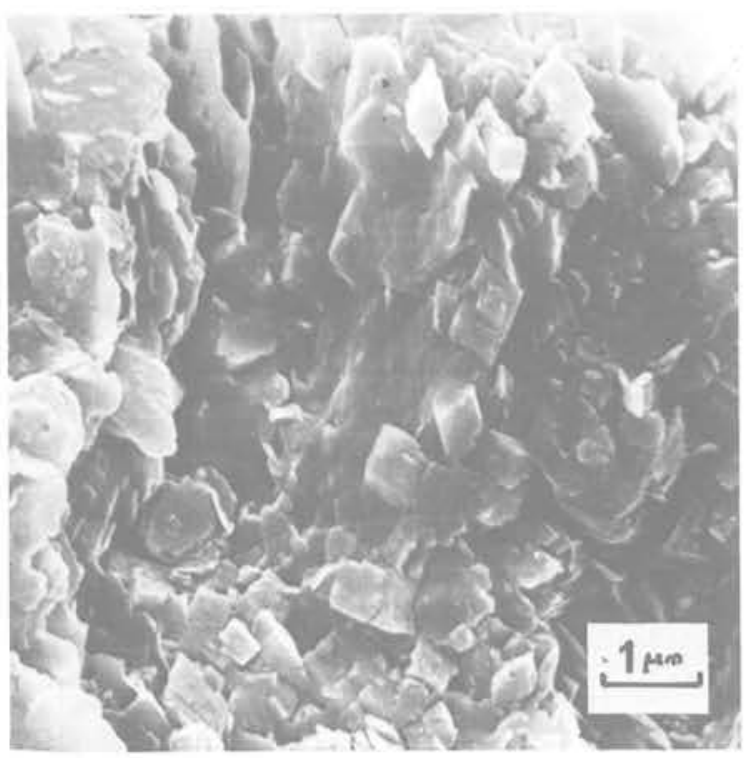

Photo 5

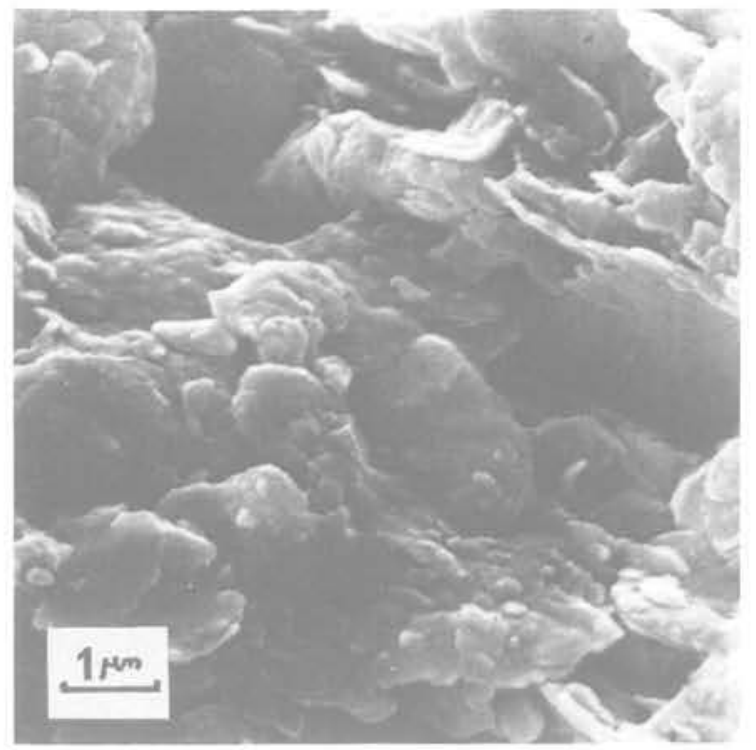

Photo 2

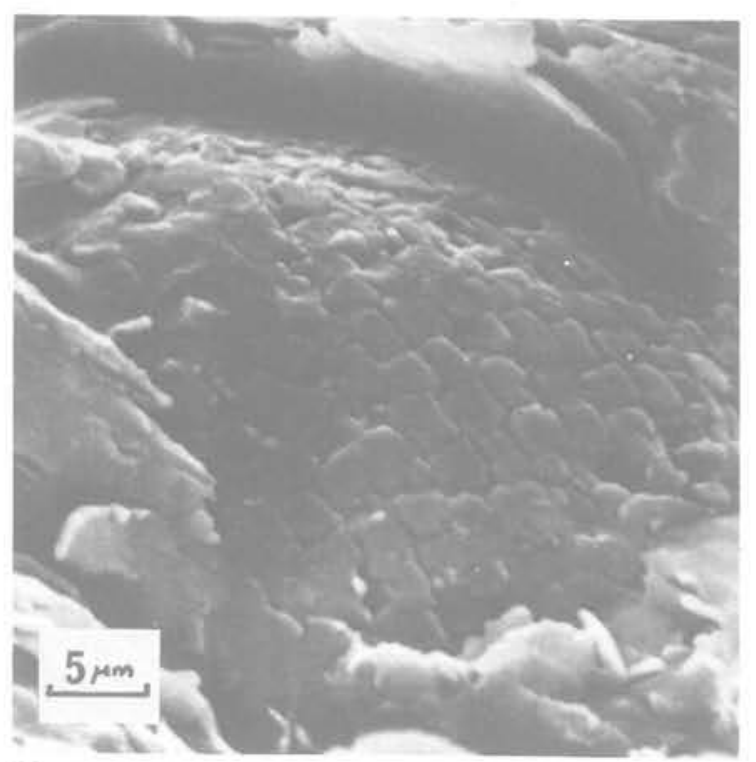

Photo 4

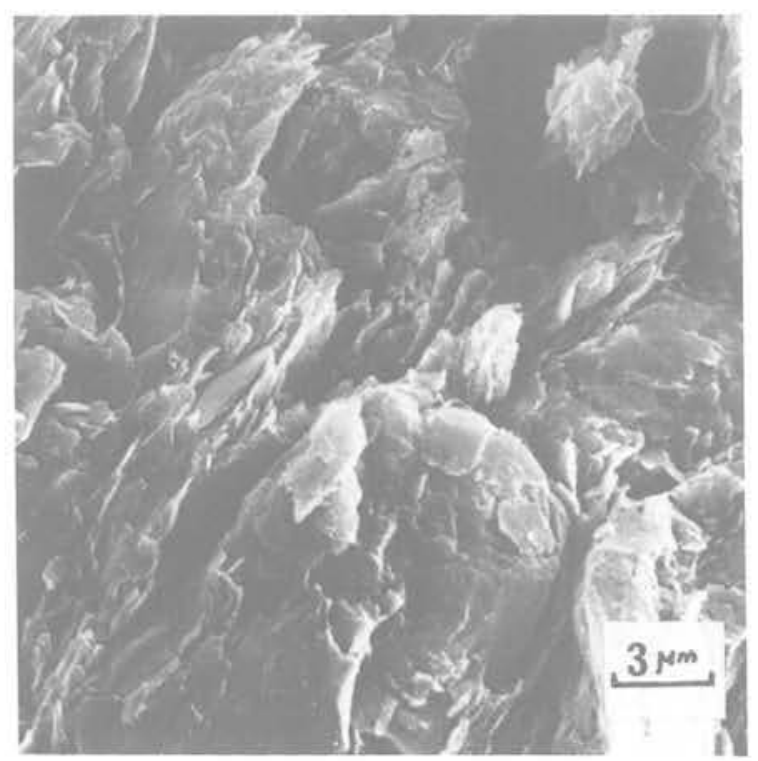

Photo 6 
Le type de liaison interparticulaire le plus fréquent dans les argilites surconsolideees du Domérien est la liaison argile/argile (A/A), c'est-à-dire une liaison majeure illite/illite. Au M.E.B., cette liaison apparait comme forte et elle sera d'autant plus forte que le matériau aura subi une consolidation élevée au cours de son histoire (8), (3). Les feuillets argileux sont étroitement emboîtés les uns dans les autres et les surfaces de contact sont très déformées. Ce type de liaison semble résister aux cycles séchage/mouillage mais est détruit lors des essais d'Atterberg (niveau 1 , tableaux I et II, photographie 1).

Le second type, également fréquent, est la liaison interparticulaire argile/micrite (A/M) (photographie 2). II convient de rattacher aux liaisons interparticulaires cette seule liaison $\mathrm{A} / \mathrm{M}$ (illite/micrite). Elle est forte (M.E.B.) et n'est pas détruite par les cycles séchage/mouillage. Elle résiste bien aux efforts lors des essais d'Atterberg (niveau 5, tableaux I et II). II existe également une liaison argile/bioclasts (A/B), mais celle-ci (illite/carbonate de calcium d'origine organique) est faible ou nulle. C'est une liaison type "sol". Les bioclasts sont générateurs de discontinuités ouvertes dès que leur dimension est supérieure à $80 \mu \mathrm{m}$; il y a alors absence de liaison (photographies 3 et 4). L'ouverture de ces discontinuités peut jouer un rôle secondaire vis-à-vis des liaisons $A / A$ et $A / M$ : elle favorise la pénétration de l'eau dans la texture de l'argilite et permet une meilleure "attaque" des liaisons $A / A$ et $A / M$ (altération météorique, par exemple). Cette attaque sera d'autant plus sensible que le nombre des liaisons $A / M$ sera faible.

Les autres types de liaisons interparticulaires existant dans les argilites du Domérien sont de répartition aléatoire. Ce sont des liaisons à E. L. I. faible et, en ce sens, elles peuvent être assimilées aux liaisons de type " sol ". Elles sont, en général, détruites ou neutralisées par l'eau. On peut citer la liaison argile/mica mais, dans les argilites du Domérien, elle a moins d'influence sur le comportement mécanique que les liaisons avec des minéraux gonflants ou de la pyrite. L'influence de ces liaisons est conditionnée par la texture des minéraux les constituant (8). L'E.L.I. ultime du matériau est donc étroitement dépendante de cette texture.

S'il y a en présence argile/montmorillonite, la liaison interparticulaire est assez faible quand la montmorillonite est diffuse $(A / \mathrm{md})$, faible si elle est concentrée en "nids" $(A / m n)$ (niveau 2, tableaux I et 11 , photographie 6). Ces liaisons interparticulaires $\mathrm{A} / \mathrm{md}$ ou $\mathrm{A} / \mathrm{mn}$ sont assimilables aux liaisons type "sol" et sont détruites en présence d'eau. L'effet de gonflement sera d'autant plus important que le nombre de liaisons
$\mathrm{A} / \mathrm{mn}$ sera grand et que la texture de l'argilite contiendra des liaisons $A / A$. Les liaisons $A / M$ peuvent s'opposer à cette expansion. A la limite, une liaison $\mathrm{A} / \mathrm{md} / \mathrm{M}$ possède une E. L.I. assez forte, le pourcentage global de montmorillonite dans les argilites restant toujours faible.

Le cas, plus fréquent, des liaisons argile/pyrite diffuse $(A / P)$ ou argile/pyrite concentrée $(A / p c)$, est analogue à celui des liaisons $A / m d, A / m n$. La liaison $A / P$ est faible et sera d'autant plus faible que le grain de pyrite sera de dimension élevée. La liaison $A / p c$ (pyrite en "colonisation" de bioclasts, par exemple; photographies 4 et 5) est faible ou nulle. Elle est équivalente à la liaison A/B, la nature du bioclast étant seule modifiée. Ces liaisons $A / P$ ou $A / p c$ ne résistent pas à l'eau et l'influence secondaire du gonflement des pyrites par oxydation en sulfates sera d'autant plus importante que le pourcentage de pyrite concentrée sera élevé (5), (8)

Les liaisons interparticulaires $\mathrm{A} / \mathrm{md}, \mathrm{A} / \mathrm{mn}, \mathrm{A} / \mathrm{P}$ et $\mathrm{A} / \mathrm{pc}$ ne doivent pas être négligées. Elles illustrent bien le rôle de la texture d'un matériau vis-à-vis de son comportement mécanique.

En définitive, on s'aperçoit que les argilites des niveaux 1 et 3 sont relativement homogènes (voir les paramètres portés dans les tableaux I et II) : elles possêdent une texture homogène constituée par un mélange illite/micrite. Les liaisons interparticulaires sont de type $\mathrm{A} / \mathrm{A}$ et $\mathrm{A} / \mathrm{M}$, mais les pourcentages relatifs entre $A / A$ et $A / M$ varient entre les niveaux 1 et 3 . Ces liaisons résistent bien aux cycles séchage/mouillage mais sont détruites lors des essais d'Atterberg. Dans les niveaux 4 et 5 , les liaisons sont encore des liaisons $A / A$ et $A / M$, mais les liaisons $A / B$ et $A / p c$ sont assez nombreuses. La texture induite par ces liaisons est plus facilement pénétrée par l'eau (discontinuités ouvertes) et l' " attaque n des liaisons interparticulaires est plus vigoureuse : il y a fragmentation du matériau. Cependant, si les E. L.I. ultimes sont plus faibles que pour les niveaux 1 et 3 , on peut penser qu'une part importante des liaisons $\mathrm{A} / \mathrm{M}$ résiste aux essais d'Atterberg et est éliminée lors de la préparation du mortier (8) (tableaux I et 11 , exemple du niveau 5 ).

Quant au niveau 2, il pose le cas particulier d'une texture constituée de liaisons $A / A, A / B, A / m n$ et $A / p c$. Le gite particulier des pyrites et de la montmorillonite favorise l'expansion et la fragmentation du matériau en présence d'eau. II ne semble pas y avoir de liaisons $\mathrm{A} / \mathrm{M}$ nombreuses et susceptibles de s'opposer à l'expansion.

En résumé, il est possible de dresser le tableau ci-après :

Tableau III - Les différents types de liaisons interparticulaires des argilites du Domérien de Jeandelaincourt, E.L.I. et propriétés.

Type de la liaison

argile/argile

argile/micrite

argile/mica

argile/bioclast

argile/montmo-

rillonite diffuse

argile/montmo-

rillonite en " nids"

argile/pyrite diffuse

argile/pyrite

en concentration
Symbole E.L.

A/A

$\mathrm{A} / \mathrm{M}$

A/B

$\mathrm{A} / \mathrm{mn}$

A/P

$\mathrm{A} / \mathrm{pc}$

assez forte

forte

faible

nulle

faible

faible

nulle assez forte sensible à l'eau

très sensible à l'eau sensible à l'eau

Propriété

sensible à l'eau

peu sensible à l'eau

sensible à l'eau

très sensible à l'eau

très sensible à l'eau 


\section{Conclusion}

Les seuls essais d'identification physique et géotechnique ne permettent pas de prévoir le comportement mécanique des argilites, même si l'on a conclu préalablement à une identité de nature et d'homogénéité de ces matériaux.

L'étude de la répartition spatiale des minéraux constitutifs et sa corrélation à des essais simples de désagrégation par cycles mouillage/séchage, conduit à la compréhension de certains comportements mécaniques. Le microscope électronique à balayage est un outil fondamental dans cette recherche puisqu'il permet la reconnaissance, I'identification et l'interprétation des textures et des liaisons interparticulaires résultant de l'histoire subie par les argilites. Les liaisons créées par les carbonates de calcium sont fondamentalement différentes s'il s'agit de carbonates d'origine chimique ou ceux d'origine biochimique. Le comportement des matériaux vis-à-vis de l'eau sera influencé par ces liaisons interparticulaires, mais également par la présence - en un gîte particulier de minéraux type pyrite ou montmorillonite.

Le rôle de la répartition spatiale des constituants est bien montré par ces aspects ; les énergies de liaisons interparticulaires sont donc dues, d'une part à I'histoire du matériau (diagenèse, consolidation, etc.), mais aussi à la manière dont s'agencent les liaisons. II devient donc obligatoire de caractériser les liaisons interparticulaires tant par leur nature que par leur géométrie. L'influence de la liaison interparticulaire argile/micrite sur les essais d'Atterberg est un exemple frappant (8).

La méthode proposée pour évaluer l'énergie de liaison interparticulaire d'une argilite surconsolidée est simple: il s'agit d'une estimation au sens propre du terme. Le pourcentage relatif entre les énergies de liaisons interparticulaires majeures $(A / A, A / M)$ peut être obtenu en éliminant de FG la fraction bioclast et la fraction pyrite, puis en dosant les pourcentages en micrite et en argiles.

A la limite, la connaissance par le M.E. B. des textures des argilites du Domérien permet dorénavant de s'affranchir de l'étude au M. E. B., de mesurer E. L. I. sur la fraction FG et de doser uniquement la micrite. II est évidemment impensable de ne pas étudier les textures et les types de liaisons au M.E.B. si le matériau argileux surconsolidé est «inconnu », l'analyse au M.E.B. permettant la mise au point de techniques d'étude complémentaire.

II serait maintenant souhaitable d'observer l'évolution des liaisons interparticulaires $(A / M$ et $A / A)$ lorsque la nature de l'assemblage argileux varie. L'étude proposée sur le Domérien ne met essentiellement en rapport que des liaisons "illite/illite" ou "illite/micrite",

\section{Références bibliographiques}

(1) Allouc J. (1977). - Contribution à l'étude sédimentologique du Pliensbachien de la région de Nancy (bordure orientale du Bassin Parisien).

Thèse présentée à l'Université de Nancy I, soutenue le 8 décembre 1977 pour obtenir le grade de Docteur de $3^{\circ}$ cycle.

(2) Bjerrum L. (1967), - The third Terzaghi lecture : progressive failure in slopes of overconsolidated plastic clay and clay shales.

Journal of the Soil Mechanics and foundations Division. Proceedings of the A. S. C. E., vol. 93, n SM5, part 1. Ann Arbor Mich. U.S.A., septembre 1967, p. 11-23.

(3) Le Roux A. (1972), - Caractéristiques mécaniques des roches argileuses en relation avec leur structure.

Bulletin de liaison des Laboratoires des Ponts et Chaussées. Laboratoire Central des Ponts et Chaussées, septembre-octobre 1972, p. 155-178.

(4) Plaza Nieto G. (1980). - Comportement d'argiles raides fissurées soumises à des essais de chargement cyclique.

Mèmoire présenté à l'Institut National Polytechnique de Lorraine, soutenu le 14 octobre 1980 pour obtenir le Diplôme d'Études Approfondies.

(5) Thomas A. (1975). - Principes et méthodes de valorisation de l'information en mécanique des sols appliquée.

Thèse présentée à l'institut National Polytechnique de Lorraine, soutenue le 28 janvier 1975 pour obtenir le grade de Docteur ès Sciences.

(6) Tisot J.-P. et Troalen J.-P. (1980). - Description et propriétés géotechniques d'argilites et marnes de Lorraine.

Compte rendu des Journées Universitaires "Génie Civil ", Institut National des Sciences Appliquées de Lyon, 22-23 octobre 1980.

(7) Tisot J.-P., Troalen J.-P. et Houpert R. (1981). Comportement d'une argile raide fissurée sous chargement cyclique.

Compte rendu action D.G.R.S.T. $n^{\circ} 78.7 .0242$, juin 1981.

(8) Troalen J.-P. (1978). - Propriétés physiques et géotechniques des argiles fissurées du Domérien de la région de Nancy.

Thèse présentée à l'Institut National Polytechnique de Lorraine, soutenue le 25 juillet 1978 pour obtenir le grade de Docteur de $3^{\circ}$ cycle.

(9) Vigouroux A. (1977). - Propriétés géomécaniques des argiles raides fissurées. Cas des argiles du Domérien moyen de Lorraine.

Thèse présentée à l'Institut National Polytechnique de Lorraine, soutenue le 7 juillet 1977 pour obtenir le grade de Docteur de $3^{\circ}$ cycle. 
